\title{
A Rapid Blood Test for Early Detection of Sepsis
}

\author{
Francis HC Tsao* and Keith C Meyer \\ Division of Allergy, Pulmonary and Critical Care Medicine, Department of Medicine, School of Medicine and Public Health, USA
}

*Corresponding author: Francis HC Tsao, Division of Allergy, Pulmonary and Critical Care Medicine, Department of Medicine, School of Medicine and Public Health, USA

\begin{tabular}{lll}
\hline ARTICLE INFO & & ABSTRACT \\
\cline { 1 - 1 } $\begin{array}{l}\text { Received: } \\
\text { Published: November 25, } 2019\end{array}$ & $\begin{array}{l}\text { Abbreviations: ICUs: Intensive Care Units; CDC: Centers for Disease Control and } \\
\text { Prevention; sPLA2: Secretory Phospholipase A2; SFA: Specific Fraction of Albumin; FFA: }\end{array}$ \\
\hline
\end{tabular}

Citation: Francis HC Tsao, Keith C Meyer. A Rapid Blood Test for Early Detection of Sepsis. Biomed J Sci \& Tech Res 23(3)2019. BJSTR. MS.ID.003909.

\section{Introduction}

Sepsis is a dysregulated host response to infection that causes organ dysfunction and circulatory hypoperfusion. It is mainly triggered by infection and occurs when a systemic inflammatory response to infection becomes overwhelming and causes cell death and tissue damage that leads to organ system dysfunction. Sepsis is the leading cause of death for hospitalized patients and entails major costs for patients and health care providers. Children and adults admitted to Intensive Care Units (ICUs) for treatment of serious infections are particularly vulnerable to infectiontriggered sepsis. Because a fatal outcome is highly likely if sepsis is not identified and treated early, the Centers for Disease Control and Prevention (CDC) has classified sepsis as a medical emergency requiring early recognition and prompt intervention. Although early diagnosis of sepsis is critical to minimize patient morbidity and mortality, a blood test that can be used to quickly and reliably diagnose early sepsis has yet to be validated.

In light of the urgency to detect sepsis in its earliest stages, two serum fluorescent assays that we previously developed could be useful for the early diagnosis of infection-triggered sepsis. These assays are sensitive, inexpensive, easily performed and can be completed within 30 minutes. One assay can detect and quantitate the infection-induced, inflammatory response secretory phospholipase A2 (sPLA2) activity in serum [1], while the second assay can measure the functional activity of a specific fraction of albumin (SFA) in the serum [2]. We have shown that the SPLA2 assay accurately measures the serum $14 \mathrm{kDa}$ protein, sPLA2 activity in an animal model [3]. When rats were challenged with endotoxin, sPLA2 increased in a time-dependent manner to the maximum level 24-48 hours following endotoxin exposure and then gradually returned to baseline levels as recovery occurred [3]. However, elevation of sPLA2, which is an acute phase protein, is also associated with non-infectious acute phase responses such as in post-operative settings [4]. Therefore, like many acute phase proteins in the serum, measuring the blood level of sPLA2 alone cannot reliably identify infection-triggered sepsis. This lack of specificity when using sPLA2 measurements to detect sepsis can be enhanced if our second assay that measures SFA is combined with measuring sPLA2.

Infection may trigger an oxidative stress response in the body with an imbalance between the increased production of free radical oxygen species and limited antioxidant defenses. Albumin is the most abundant and important antioxidant in circulating blood, and serum albumin has multiple functional activities that include the ability to bind fatty acids (FA), certain hormones, metal ions, drugs and nutrients and transport these throughout the body to support cellular function. Additionally, albumin can also act as a scavenger to bind and remove breakdown products of cellular membrane phospholipids (PL) to maintain cell membrane homeostasis [2]. Free fatty acids (FFA) and lysophospholipids (lysoPL) are the two major breakdown products derived from membrane PL. We have found that pre-binding of FFA and lysoPL to albumin depleted albumin-binding capacity in a FFA/lysoPL dose-dependent manner, 
and this capacity was completely depleted if albumin was saturated with pre-bound FFA/lysoPL [2].

The SFA assay is a real-time measurement of a specific fraction of albumin binding capacity with bilayer membrane constituents, and the SFA assay can be used as a quantitative measurement to assess the depletion of albumin binding capacity for cell membrane derived FFA and lysoPL. When using the SFA assay, we found that the SFA activity was completely depleted in the serum samples collected from three patients with sepsis at the time of admission to the ICU [2], and SPLA2 activity was elevated in the sera of these septic patients [1]. We have also studied an additional cohort of septic patients and found that sPLA2 levels and SFA values gradually normalize if patients recover from their illness [5].

Because the quantity of serum albumin from critically ill, septic patients was only about $30 \%$ lower than the normal level for healthy subjects, total depletion of SFA activity strongly suggests pre-binding of FFA/lysoPL to albumin in the sera of the septic patients saturates binding sites and prevents further binding. We speculate that the saturation of albumin with pre-bound FFA/ lysoPL was likely due to scavenging of degraded PL derived from disrupted cell membranes of dead and dying cells by albumin and reflects tissue and organ damage caused by a sepsis-triggered systemic inflammatory response [3]. The information described above is summarized in Figure 1. We have also assayed a large number of serum samples collected from clinically stable patients with chronic obstructive pulmonary disease $(n=5)$, cystic fibrosis $(n=7)$, or idiopathic pulmonary fibrosis $(n=26)$. None of these samples had elevated SPLA2 activity, the SFA activity in these serum samples was decreased by only $20-30 \%$ of the normal values found in healthy subjects, and no serum sample from non-septic subjects had complete depletion of SFA. These results suggest that the SFA assay, when combined with the sPLA2 assay to generate a SPLA2/ SFA ratio, may prove useful as a rapid test to detect early stages of sepsis in at-risk patients suspected to have serious infections. Additionally, the sPLA2/SFA ratio may be useful to assess the efficacy of therapeutic interventions over time. However, serial sampling of multiple serum samples from a large cohort of patients with suspected infection who are at significant risk to develop sepsis are needed to corroborate the results shown in Figure 1 and validate the potential use of the two-component SPLA2/SFA assay as a clinically useful tool for patient care.
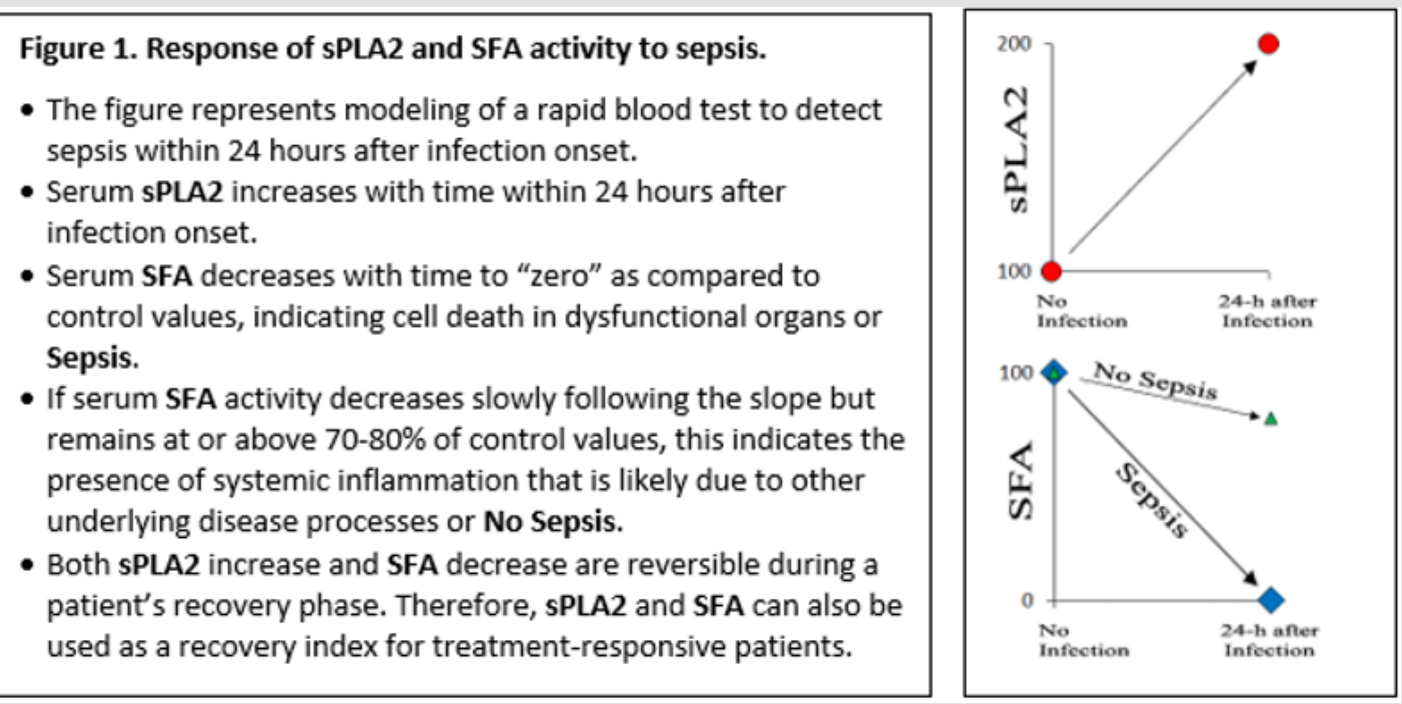

Figure 1: Response of Spla2 and SFA activity to sepsis.

\section{References}

1. Tsao FHC, Shanmuganayagam D, Zachman DK, Khosravi M, FoltsJD, et al. (2007) A continuous fluorescence assay for the determination of calcium-dependent secretory phospholipase A2 activity in serum. Clin Chim Acta 379(1-2): 119-126.

2. Tsao FHC, Xiang Z, Meyer KC (2015) Fluorescent Determination of Secretory Phospholipase A2 (sPLA2)-Mediated Human Serum Albumin Binding Activity with Membrane Phospholipids and Fatty Acids. Translational Medicine 5: 1-9.

3. Tsao FHC, Culver BJ, Pierre JF, Shanmuganayagam D, Patten CC, et al (2012) Prophylactic supplementation of grape polyphenolics attenuates endotoxin-induced serum secretory phospholipase A2 Activity in rats. Comp Med 62: 271-278.

4. Ogawa M, Arakawa H, Yamashita S, Sakamoto K, Ikei S (1992) Postoperative elevations of serum interleukin 6 and group II phospholipase A2: group II phospholipase A2 in serum is an acute phase reactant. Res Commun Chem Pathol Pharmacol 75(1): 109-115.

5. Meyer KC, Tsao FHC, Amessoudji AW, Jawdat D, Sadat M, et al. (2017) Determination of a Serum-Specific Fraction of Albumin Binding Activity Mediated by a Secretory Phospholipase A2 as a Sepsis-Specific Biomarker. Chest 152(Supplement 4): a409. 
ISSN: 2574-1241

DOI: 10.26717/BJSTR.2019.23.003909

Francis HC Tsao. Biomed J Sci \& Tech Res

(C) This work is licensed under Creative

Submission Link: https://biomedres.us/submit-manuscript.php

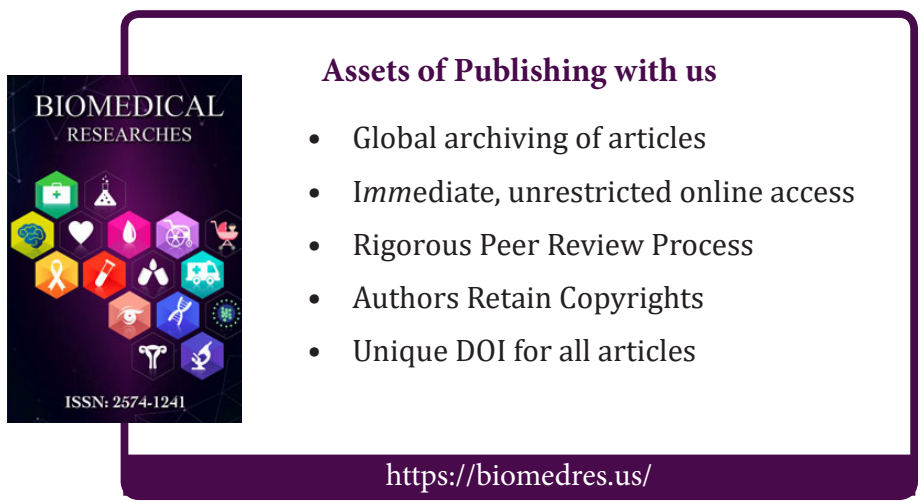

